\title{
Considerations on the Licensed Shared Access (LSA) Architecture from the Incumbent Perspective
}

\author{
Miia Mustonen ${ }^{1}$, Marja Matinmikko ${ }^{1}$, Marko Palola ${ }^{1}$, Seppo Yrjölä ${ }^{2}$, Jarkko Paavola ${ }^{3}$, Arto Kivinen ${ }^{3}$, Jan Engelberg ${ }^{4}$
}

\begin{abstract}
As the amount of mobile data continues to grow rapidly, finding frequency bands without incumbent usage for mobile network operators (MNOs) in a harmonized manner becomes increasingly challenging. This motivates the search for new methods to increase the efficiency of current spectrum usage by exploring the new possibilities to allow multiple services to share the same frequency band in a controlled manner. This kind of spectrum sharing should be conducted in a way that requires minimum changes to the functionalities and the architecture of the systems involved and especially to the current holders of the spectrum usage rights, thus, the incumbent spectrum users. In this paper, we concentrate on the emerging Licensed Shared Access (LSA) concept which is currently receiving a lot of attention in European regulation and standardization. We discuss different types of incumbents and the critical factors for allowing spectrum sharing with LSA from their perspective. Finally, we propose an architecture for LSA that takes into account these critical factors while guaranteeing certain quality of service $(\mathrm{QoS})$ conditions for all involved systems.
\end{abstract}

Keywords-LSA, Licensed Shared Access, ASA, Authorised Shared Access, spectrum sharing, cognitive techniques

\section{INTRODUCTION}

Shared use of spectrum has received a lot of attention as the rapidly increasing amount of mobile data motivates the search for new innovative ways to increase the efficiency of the spectrum use. For mobile network operators (MNOs), techniques for spectrum sharing become critical as not even all of the bands that have recently been allocated for International Mobile Telecommunications (IMT) are available for them on an exclusive basis in a harmonized manner globally.

In the current spectrum regulatory framework, frequency bands are in general exclusively licensed to a single entity or license-exempt with open access to all [1]. A lot of attention in research [2] has been paid to different forms of opportunistic use of spectrum usage: a license-exempt approach where an entity uses its capabilities to autonomously find a spectrum opportunity to transmit data. An example of such approach is a cognitive radio system based on spectrum sensing [3]. However, from a regulatory point of view allowing a system to use spectrum in an uncontrolled manner is difficult due to interference considerations. Another license-exempt approach is collective use of spectrum (CUS) in which a designated spectrum band is opened for an unlimited number of independent users to access it at the same time and in a particular geographic area under a well-defined set of conditions [4]. One practical example of the CUS approach is the TV white space usage [5] where so called white space devices (WSD) are allowed to access parts of the TV band in a

${ }^{1}$ VTT Technical Research Centre of Finland, Oulu, Finland,

firstname.lastname@vtt.fi. license-exempt manner while protecting incumbent spectrum use. However, this approach has not created business for MNO since the main problem with the CUS approach in general is that it does not guarantee interference-free operation or resource availability.

In order to complement the two existing regulatory approaches, namely the licensed and license-exempt approaches, new concepts emerging from industry and research have been introduced into spectrum regulation. In U.S, a threetier regulatory model introduces three different hierarchy levels of licenses. In this approach a device is allowed to use a spectrum band if a database does not include a conflicting higher order license user [6]. Also a European research driven pluralistic licensing model considers different levels of licenses and sharing conditions [7]. In Licensed Shared Access (LSA) approach, an incumbent shares its spectrum with a limited amount of LSA licensees in a way that interference is avoided [8]. The LSA approach is receiving growing attention as a potential means for speeding up the process of making IMT bands available for MNOs in a timely manner to respond the market growth.

The focus of this paper is on LSA which has rapidly gained a lot attention in European regulation and standardization activities. As a current holder of spectrum usage rights the incumbent is vital for successful introduction of LSA. There is no prior work addressing specifically the incumbent role in LSA. Therefore in this paper, we introduce incumbent types and usage scenarios and propose an architecture for the LSA approach that takes into account the critical factors from incumbent perspective. The rest of the paper is organized as follows. Section II introduces the LSA concept. The current status of LSA architecture is reviewed in Section III. In Section IV, different incumbent types and spectrum usage scenarios are introduced. Based on these, the critical factors and the resulting enhanced architecture are proposed in Section V. Finally, conclusions are drawn in Section VI.

\section{LSA CONCEPT}

LSA concept was initially introduced by European Commission (EC) as means to enable more efficient use of spectrum by allowing two or more radiocommunication systems to share the same spectrum band in a fully controlled manner [4]. LSA is defined by the EC as "A regulatory approach aiming to facilitate the introduction of radiocommunication systems operated by a limited number of licensees under an individual licensing regime in a frequency band already assigned or expected to be assigned to one or 
more incumbent users. Under the Licensed Shared Access (LSA) approach, the additional users are authorised to use the spectrum (or part of the spectrum) in accordance with sharing rules included in their rights of use of spectrum, thereby allowing all the authorized users, including incumbents, to provide a certain Quality of Service $(Q o S)$ " [8]. In practice, this means that individual LSA licenses with limited duration and/or area will be issued to permit additional usage on spectrum bands that are currently being underutilized by other type of incumbents.

The LSA concept is an extension of an industry driven Authorised Shared Access (ASA) concept which was proposed as a solution to ensure harmonised spectrum for mobile broadband in the bands identified for IMT. ASA was assessed in the Working Group Frequency Management (WG FM) of European Conference of Postal and Telecommunications Administrations, the Electronic Communications Committee (CEPT ECC) [9]. The first live demonstration of the ASA concept was shown in Finland in April 2013 and reported in [10]. Even if LSA is a wider concept introducing shared use of spectrum bands, not limited only to the mobile broadband or IMT bands, this will be the first application area of LSA. The key benefit arising from LSA concept is that it enables timely availability of harmonized spectrum with predictable QoS for both existing and new users called the incumbents and LSA licensees, respectively.

The LSA concept is based on an individual LSA license and the related sharing framework negotiated between the key players involved in LSA on a voluntary basis: the LSA licensee, the incumbent and the regulator [1]. The sharing framework determines rules and conditions under which the LSA licensee may use the spectrum band. In LSA, the rights of the incumbent are preserved and the resulting LSA band availability for sharing with an MNO is allowed to change according to the incumbent activity in the spectrum band e.g. in a certain geographic area due to an unexpected need for the resource. The sharing framework may e.g. determine the timeframe on which the LSA licensee (MNO) needs to evacuate the specified spectrum band. The LSA concept does not exclude any technologies such as cognitive radio, which may have a role as enablers for sharing under the LSA approach.

In Europe, LSA is under study both in regulation and standardization. In regulation, there are currently two project teams under CEPT ECC WG FM that are conducting studies on LSA: PT FM53 is investigating the general concept [1] and PT FM52 is concentrated on the application of LSA concept for MNOs in the 2.3-2.4 GHz band in time division duplexing (TDD) mode. In standardization, European Telecommunications Standards Institute (ETSI) has developed a system reference document describing mobile broadband services in 2.3-2.4 GHz band using LSA [11]. The work has been continued by specification of the system requirements. After these, functional architecture and protocols will be developed.

\section{REVIEW OF CURRENT LSA ARCHITECTURE}

Any decisions on LSA architecture have not been made yet in ETSI RRS, however, some discussion on the components of the technical realization have been discussed already in [11]. Additionally, some discussion on the functional blocks for implementation of LSA has been conducted in CEPT [1].

An overview of the proposed LSA architecture is shown in Figure 1. As an initial step, the LSA license and the sharing framework is negotiated between the LSA licensee, the incumbent and the regulator. These agreements form a basis for the sharing. The sharing framework contains high level rules for sharing and may include items such as protection criteria for the incumbent, procedure for reclaiming a spectrum band and the required evacuation time, possible compensation for spectrum usage etc. Both the incumbent and the LSA licensee will make the decision on whether to share the spectrum using LSA based on the developed sharing framework. To allow operational certainty, the sharing framework should remain unchanged throughout the LSA license duration.

The management of time and area dependent LSA spectrum resources between the key players of LSA adds an additional spectrum management layer on top of existing cellular systems shown as LSA Management System in Figure 1. Currently it is foreseen to contain two management units, namely the LSA repository and LSA controller [11]. The LSA repository is a database that contains up-to-date information on the available LSA bands and the LSA controller controls the access to the LSA spectrum and configures the network parameters accordingly. The LSA repository may serve and coordinate between multiple LSA controllers and it ensures that the LSA band information is available only to the relevant LSA controller. In our work, we consider the LSA controller to be fully inside the domain of the MNO's network to help protect the confidential information of the MNO network required for the configuration process. In practice, the LSA architecture is an open topic and the final LSA architecture may divide the tasks of different management units also in different manner.

\section{INCUMBENT TYPES AND SPECTRUM USAGE SCENARIOS IN LSA}

Under LSA approach, an incumbent refers to a current holder of spectrum rights of use [8]. Their role is critical in LSA in order to create a sharing framework that offers attracting operational conditions for both the incumbent and the LSA licensee. Thus for the successful introduction of the LSA concept into real-life systems, the incumbents' rights need to preserved but the protection criteria should not overly restrict the operations of the MNO. In the following, we introduce different types of incumbents and their usage scenarios. 


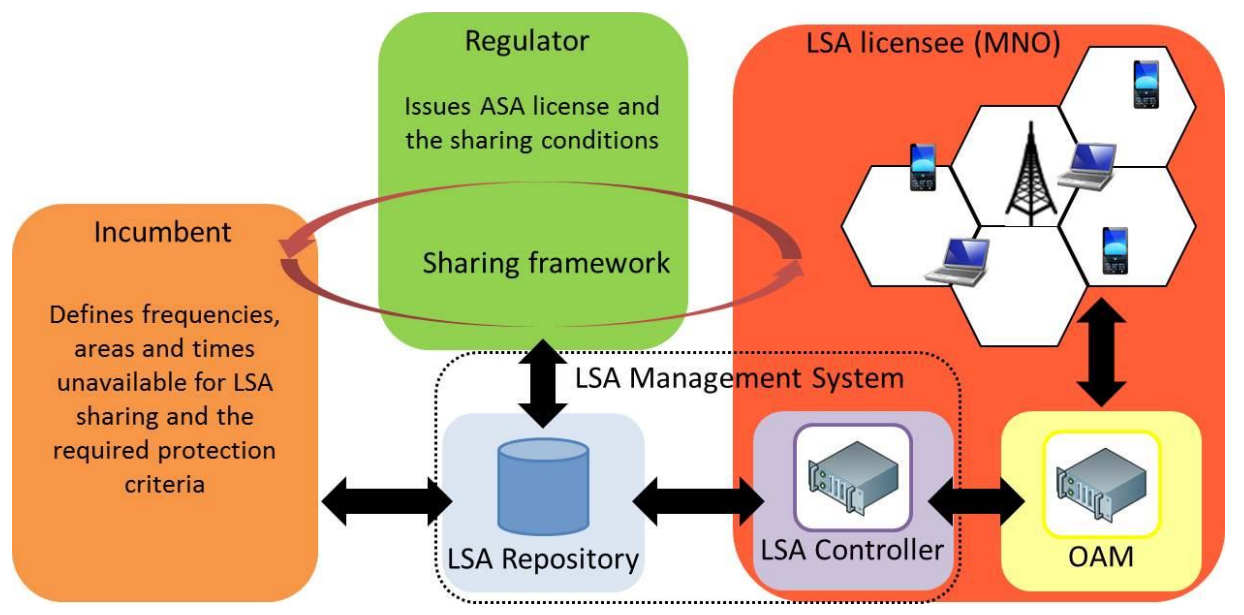

Figure 1. LSA Architecture

\section{A. Governmental vs. Commercial Incumbent}

On the basis of the spectrum rights of use, incumbents can be divided into two categories: governmental and nongovernmental. Governmental incumbents have access to frequency bands to perform their own duties but they do not have individual rights of spectrum use. Their access to a band is usually on a shared basis and regularly reviewed by the administration. A governmental incumbent can cover various domains such as defense, public safety, meteorology and science [1]. A commercial or non-governmental incumbent refers to a private entity that the administration has granted spectrum usage rights to. The rights can be either individual or general. The individual rights of spectrum use (or an individual license) is usually granted for a limited period of time and it includes rights and obligations for the licensee e.g. obligation on coverage for MNOs [1].

The main difference between the two types of incumbents with respect to the introduction of LSA is that since the governmental user does not possess individual usage rights and the access on a band may be on a shared basis, introducing additional users with LSA is more straightforward. Individual rights of spectrum use provide commercial incumbent protection against obligations to share until the end of the license period. It is therefore currently anticipated that the LSA concept would be most beneficial when applied to a spectrum band with a governmental user as an incumbent. In practice, LSA concept is better applicable to a case where the incumbent user and the LSA licensee are of different kind [8].

\section{B. Dynamic vs. Static Spectrum Usage}

Additionally, incumbents can be divided into dynamic and static based on their spectrum usage patterns. Dynamic spectrum usage is not tied to a particular geographical place or to a certain time. The usage varies even over a short period of time without a predictable pattern. An example of an incumbent with dynamic spectrum usage is a broadcasting service provider with mobile wireless camera and video links. For LSA, an incumbent with dynamic spectrum usage requires dynamic management functionalities and the importance of LSA repository and LSA controller is highlighted.

Static spectrum usage is most of time bound to a certain geographical place or a specific time. An example of an incumbent with static spectrum usage on a certain geographical place is a military base. For LSA, interference to a static incumbent may be mostly prevented with traditional methods such as exclusion zones and times. In the LSA, the protection criteria for static spectrum usage can already be defined in the sharing framework. However, LSA repository and LSA controller are still needed to report the changes in the LSA spectrum availability e.g. for incumbent to reclaim spectrum bands in the case of an emergency as well as for coordination between incumbents and LSA licensees.

In practice, the spectrum usage of an incumbent may have both static and dynamic components as shown in Figure 2. For example defense, as an example of a governmental incumbent, may have both a military base on a certain location and radars with variable location. Also a commercial incumbent e.g. a broadcasting service provider may have both a TV studio on a fixed location and mobile wireless camera and video links which change position dynamically.
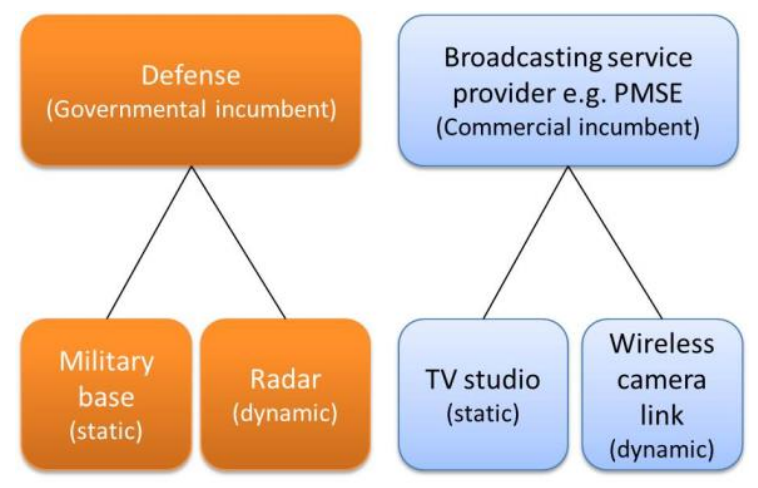

Figure 2. Classification of incumbents. 


\section{CRITICAL FACTORS AND THEIR EFFECT ON LSA ARCHITECTURE}

\section{A. Critical factors for the incumbent}

The successful introduction of LSA concept requires that there is an incentive for sharing for all key players involved. Potential benefits for MNO include capacity boost, coverage improvement, and QoS enhancement of end users [12]. For the incumbent the LSA may offer e.g. possibilities for flexibility and scalability in spectrum use as well as sharing the infrastructure. The incumbent may also be motivated for LSA by maintaining spectrum access in a longer term or by compensation for sharing [1]. In addition to benefits, also the critical factors related to sharing need to be identified from different stakeholder's view. In this paper, we focus on the incumbent perspective since the incumbent as a current holder of spectrum rights of use has to adapt its functionalities to the new, shared operation. Therefore, the prerequisite for successful roll out of LSA is that the introduction to a spectrum band is done as straightforward as possible to the incumbent. Such critical factors are discussed in the following.

\section{1) Reclaiming the spectrum band}

The core of the LSA concept lies in the fact that the incumbent is allowed to reclaim its spectrum band also during the LSA license duration in case of a need for additional spectrum. The incumbent reclaims a spectrum band by informing the LSA repository of the change in the LSA band availability by sending an evacuation request. For static spectrum use, an evacuation request is a result of an unexpected event, whereas, for dynamic spectrum usage evacuation requests are part of normal operation since all spectrum use is unscheduled and reserved using evacuation requests. Especially in this case, it is important that the incumbent can send the evacuation request in a simple manner and via different devices such as mobile phone, internet etc. Depending on the incumbent and their protection criteria, different constraints can be placed on the evacuation time as well as on the size of the area to be evacuated. In case of a governmental incumbent such as defense, a specific requirement may arise on evacuation of a spectrum band in case of a disaster.

\section{2) Maintain normal operation}

One important aspect for the incumbent is that it can maintain its normal operation regardless of sharing. In addition to informing the LSA repository on changed conditions regarding the shared band and LSA license, there should not be additional constraints or restrictions on the incumbent. This means e.g. provision of predictable QoS and support of mobility of the incumbent. For example, a TV truck with wireless camera links should be able to move freely without constantly contacting LSA repository on the changed coordinates. The MNO should be responsible for compliance with technical requirements obtained from the incumbent such as meeting certain interference thresholds.

\section{3) Hide real usage and network details}

The network information and the real usage of the incumbent network should be considered as classified information and be protected. The information that the incumbent needs to share with the LSA licensee should be limited to the minimum that is necessary for implementing sharing. The incumbent should not have to reveal the spectrum usage beyond the bands described in the LSA license. In the case of a governmental incumbent such as defense it might be necessary to add safety periods and areas to mask the actual usage of the spectrum. The LSA repository could generate a non-predictable protection mask on top of an incumbent usage area based on incumbent request. Also the information on incumbent's spectrum usage on LSA bands should be accessible only to that LSA licensee that the information is intended to e.g. in the case of multiple LSA licensees.

\section{4) Protection of the data}

The data transmitted by the incumbent e.g. regarding the LSA spectrum usage and the protection criteria should be protected. In practice this means that measures should be taken to secure the data transmitted to and from LSA repository as well as the data stored in it. Similarly to the factor 3) previously, it can be foreseen that the security of the data is more important to governmental incumbent such as defense than to a commercial incumbent.

\section{5) Feedback on the spectrum usage}

In some cases it may be important for the incumbent to get information and monitor where and when the shared bands are being used. However, in order to gain from the usage information the incumbent needs to develop mechanisms to process and store it. Whether there is a need for this depends heavily on the incumbent type. Monitoring the usage of the LSA spectrum is likely to happen for security reasons and it is therefore highly unlikely that a commercial incumbent would benefit from such information. However, it is likely that all types of incumbents would benefit on receiving an acknowledgement from the LSA licensee when the evacuation on spectrum band is finished and the band is again available for the incumbent. Additionally, the regulator may be informed on the usage level of the LSA spectrum. This information may be used by the regulator later on when negotiating LSA licenses.

Although most of the critical factors listed above are applicable to both governmental and commercial incumbent and cover both dynamic and static spectrum usage, some of them are emphasized to certain type of incumbent as discussed above. 


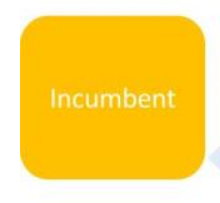

Frequency bands unavailable (time and area), incumbent type, evacuation request

Frequencies and areas being used by LSA licensee, evacuation acknowledgement
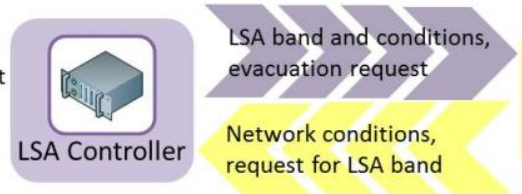

request for LSA band

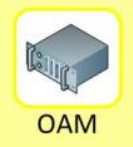

Figure 3. Information flow in LSA architecture.

\section{B. Proposed Enhanced LSA Architecture}

Especially in Europe, on a certain spectrum band there may exist a wide variety of different incumbents and they may differ significantly in different countries. For this reason, in order to ensure economies of scale, the LSA architecture should be designed in a manner that is applicable to both governmental and non-governmental incumbent types and cover both dynamic and static spectrum usage. It should therefore take into account all critical factors listed in previous subsection. The information exchange required for LSA is depicted on a high level in Figure 3. Next, the proposed functionality of LSA repository and LSA controller as well as the information flow in terms of message sequence charts are discussed in more detail.

\section{1) LSA management system and the information for LSA}

The main purpose of the LSA repository is to provide and maintain a list of available LSA frequencies. As shown in Figure 3, LSA repository receives input from the incumbent, the regulator and LSA controller of the MNO. The incumbent informs the LSA repository on the frequency bands, times and geographical areas within the LSA license that are not available for the LSA licensee. This information corresponds to the dynamic part of the incumbent's spectrum usage.

The regulator provides the LSA repository terms and conditions of the LSA license and the sharing framework negotiated between the incumbent, regulator and the LSA licensee. This information remains stable through the LSA license and therefore needs to be provided only once. The sharing framework may contain items such as the license duration, the static component of the incumbent spectrum use, the required evacuation time, protection criteria for different incumbent types etc.

Based on the information received from the incumbent and the regulator, the LSA repository creates a list of available frequencies, times and areas together with power limits which are submitted to the LSA controller of the corresponding MNO. Based on this information the LSA controller configures radio and network parameters accordingly and grants access to the spectrum band. The LSA controller ensures that the protection criteria set by the incumbent is met. As the LSA controller receives a request from the operation, administration and management (OAM) together with the network conditions, it uses that information to select and inform the OAM on the most suitable LSA band. This aspect of LSA management has been further elaborated in [12]. The LSA controller keeps the LSA repository updated on the frequencies and areas that are being used by the LSA licensee. The LSA repository makes this list available to the incumbent if so requested (see Section V A.5). Additionally, it may keep the regulator informed on the usage level of the LSA bands.

\section{2) LSA functionality}

When the information required for LSA is available at the LSA repository and the LSA controller, the LSA licensee is able to start using the LSA resources as needed as shown in Figure 4. When the OAM detects a lowered service level at some of its base stations, it sends an LSA resource request to the controller indicating the location of the base station and network conditions leading to the request [12]. The LSA controller sends a response to OAM indicating the designated LSA band for the given location, permitted transmit power and the availability period. Additionally, the LSA controller informs the LSA repository on the spectrum use by the LSA licensee. It is possible for the LSA controller to add a mask area to the location information not to reveal the exact location of the MNO base station.

In Protocol to Access White-Space (PAWS) [13] developed for license-exempt TV white space access, the resource allocation is done strictly between the white space device and the database. In LSA, however, an additional control unit is needed to provide protection on the network details of the MNO. Also in some cases there might be a need for the LSA repository to keep the incumbent up to date on the spectrum usage as discussed in Section V A.5) and shown in Figure 4.

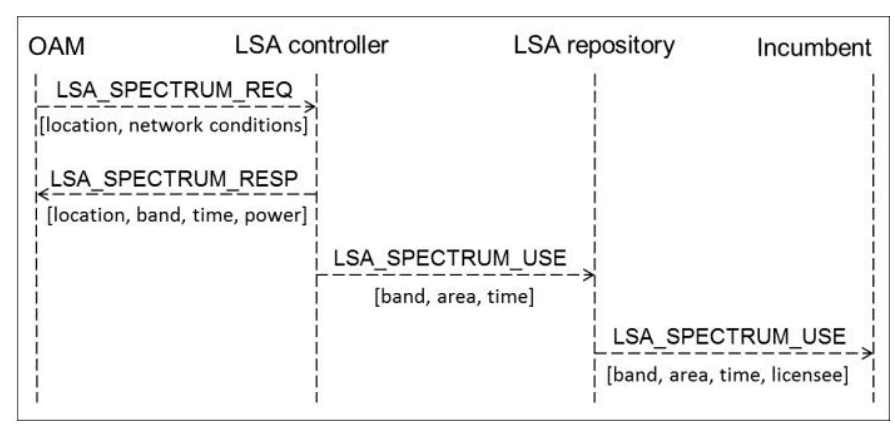

Figure 4. Request for LSA resources. 


\section{3) Evacuation request}

The incumbent informs the LSA repository on the changes in the available LSA resources in the form of an evacuation request. The evacuation request is coupled with incumbent type for the calculation of the protection area which enables interference-free operation for the incumbent. This information is then submitted by the LSA repository to the corresponding LSA controller of the MNO. The calculation of the protection area is important especially in the case of an incumbent with dynamic spectrum usage since too strict protection criteria will lead to waste of spectrum resources. The calculation of protection criteria may be done more accurately in the LSA controller where it is possible to take into account the actual surrounding of the incumbent, attenuation etc. Optionally, an incumbent may also add an indication of the expected mobility to prevent the need for frequently updating the evacuation request. After calculating the protection area, the LSA controller will pass the evacuation request to the OAM which will command the relevant base stations to stop using the affected LSA bands. The acknowledgement of the evacuation will be submitted via LSA management system back to the incumbent.

In PAWS, a WSD checks periodically the availability of the spectrum [13]. As discussed before Section V A.1), in LSA the requirement for the evacuation time depends on the incumbent and their protection criteria. In order to account also for the most critical requirements as well as to take into account both dynamic and static spectrum usage, in LSA the evacuation request is sent by the incumbent and forwarded via LSA management system to the MNO network.

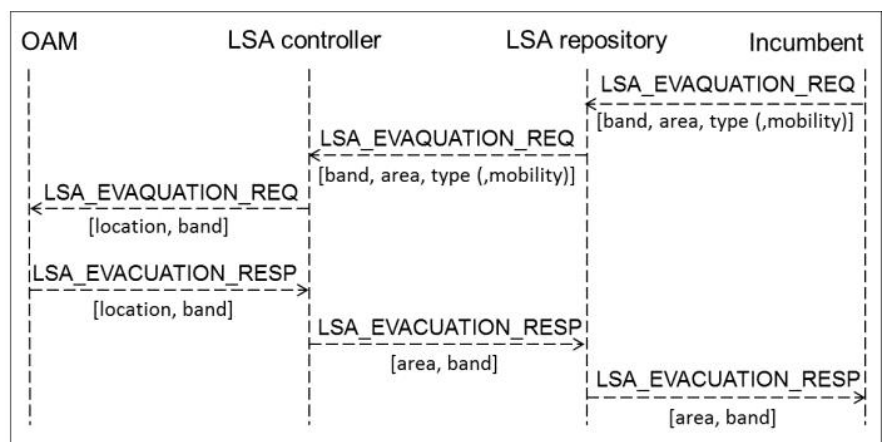

Figure 5. Evacuation request.

\section{CONCLUSIONS}

The expected explosion in the mobile data traffic in the next decades has motivated the search for new ways to use spectrum more efficiently. As finding exclusive frequency bands for MNOs in a harmonized manner is becoming increasingly challenging, effort is being placed on finding methods to share the same frequency band in a controlled manner. New concepts are arising to complement the current spectrum regulatory framework with either exclusively licensed or license-exempt spectrum bands.
In this paper, we focused on the Licensed Shared Access (LSA) concept which is currently receiving a lot attention both in European regulation and standardization activities. In the LSA, an incumbent shares its spectrum with a limited amount of LSA licensees in a way that predictable QoS can be provided for all. As the current holder of spectrum usage rights the incumbent is crucial for successful introduction of LSA, therefore in this paper we reviewed the LSA concept from the incumbent viewpoint. We introduced different incumbent types and the critical factors that need to be taken into account to ease the introduction of LSA to a spectrum band. We then proposed an enhanced architecture for LSA that takes into account these critical factors.

\section{ACKNOWLEDGMENT}

The authors would like to acknowledge CORE+ project research consortium that consists of VTT Technical Research Centre of Finland, University of Oulu, Centria University of Applied Sciences, Nokia Solutions and Networks, PehuTec, EXFO, Elektrobit, Anite, Rugged Tooling, Finnish Defence Forces, Finnish Communications Regulatory Authority, and Tekes - the Finnish Funding Agency for Innovation.

\section{REFERENCES}

[1] CEPT, "Licensed shared access", ECC Report 205, Feb. 2014.

[2] M.T. Masonta, M. Mzyece, and N. Ntlatlapa, "Spectrum decision in cognitive radio networks: A Survey," IEEE Communications Surveys \& Tutorials, vol. 15, no. 3, 2013, pp. 1088-1107

[3] I. F. Akyildiz, W.-Y. Lee, M. C. Vuran, and S. Mohanty, "NeXt Generation/Dynamic Spectrum Access/Cognitive Radio Wireless Networks: A Survey," Elsevier Computer Networks, vol. 50, May 2006, pp. 2127-2159.

[4] RSPG, "Report on Collective Use of Spectrum (CUS) and other spectrum sharing approaches", RSPG11-392, European Commission, Radio Spectrum Policy Group, Nov. 2011.

[5] CEPT, "Technical and operational requirements for the possible operation of cognitive radio systems in the 'White Spaces' of the frequency band 470-790 MHz”, ECC Report 159, Jan. 2011.

[6] PCAST, "Realizing the Full Potential of Government-Held Spectrum to Spur Economic Growth," President's Council of Advisors on Science and Technology, July 2012.

[7] O. Holland, L. De Nardis, K. Nolan etal. "Pluralistic licensing," In Proc. IEEE International Symposium on Dynamic Spectrum Access Networks (DYSPAN) 2012, pp. 33-41.

[8] RSPG, "RSPG Opinion on Licensed Shared Access", RSPG13-538, European Commission, Radio Spectrum Policy Group, Nov. 2013.

[9] ECC WG FM, "Report on ASA concept," FM(12)084 Annex 47, 2012. [Online].

http://www.cept.org//files/8694/FM(12)084\%20Annex\%2047_Report\% 20on\%20ASA\%20concept.doc

[10] M. Matinmikko, M. Palola, H. Saarnisaari etal. "Cognitive Radio Trial Environment: First Live Authorized Shared Access-Based SpectrumSharing Demonstration, " IEEE Vehicular Technology Magazine, vol. 8, no. 3, Sept. 2013, pp. 30-37.

[11] ETSI, "Mobile Broadband services in the 2300-2400 MHz frequency band under Licensed Shared Access regime," TR 103.113 v 1.1.1, July 2013.

[12] M. Mustonen, T. Chen, H. Saarnisaari etal. "Cellular architecture enhancement for supporting licensed shared access (LSA) concept," to appear in IEEE Wireless Communications Magazine in June 2014.

[13] IETF, "Protocol to Access White-Space (PAWS) Databases", The Internet Engineering Task Force, Dec. 2013. 